\title{
Optimization of silica gel beds for the use in humidity controller setups
}

\author{
Norbert Rogge ${ }^{1}$, Sven Hofmeister ${ }^{1}$, Falko Hilbrunner ${ }^{2}$, Thomas Fröhlich $^{1}$ \\ ${ }^{1}$ Technische Universität IImenau, Institute for Process Measurement and Sensor \\ Technology, \\ ${ }^{2}$ Sartorius Weighing Technology GmbH, Göttingen \\ norbert.rogge@tu-ilmenau.de
}

\begin{abstract}
The problem, discussed in this work, is the optimization of the silica gel temperature control. For fast temperature changes of the silica gel, a high density packed bed of silica gel would be necessary. But this would slow down the moisture transport in the silica gel bed due to the much faster transport in air, than on surfaces or in solid bodies.

To predict the behavior of the humidity control system, simulations were performed to preselect promising silica gel bed geometries. In Simulation of the moisture transport the nonlinear sorption isotherms of the silica gel had to be taken into account, to represent the influence of dew point itself on the dynamics of changes in dew point temperature.

The contribution deals with influences on the static and the dynamic performance of the humidity control system such as heater geometry, silica gel grain size and the microporous structure of the silica gel. Furthermore the characteristics of the test system have to be considered, to build up a model describing a humidity control system.
\end{abstract}

Key words: humidity control, silica gel, moisture transport, dew point, thermoelectric cooling

\section{Motivation}

For calibration of humidity sensors, predefined climate conditions have to be generated. An approach described in [1] uses the temperature dependent water load of silica gel to produce different humidity values continuously. This setup can be combined with an accurate humidity sensor like a dew point mirror, to generate well known states of humidity.

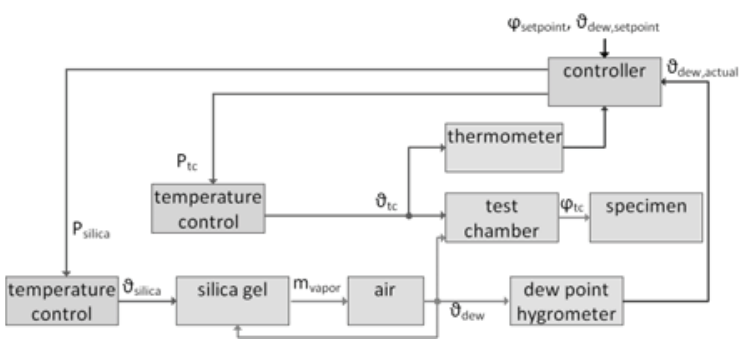

Fig. 1: Scheme of humidity control setup

A schematic example of such a control setup is shown in Fig. 1. The generation of humidity by temperature control of silica gel allows a continuous and precise setting of dew point temperatures in broad measurement range.

\section{Characteristics of thermal hygrostat}

The behavior of silica gel is described with sorption isotherms. The adsorbed amount of water $m_{\text {Water }}$ is typically given in relation to the mass $m_{S G}$ of silica gel. One form gives the water load $X$ in dependence on the relative humidity $\varphi$ or the absolute humidity $c$ :

$$
\begin{aligned}
& X(\varphi, T)=\frac{m_{\text {Water }}}{m_{S G}}=X_{m}(T) \cdot f_{r}(\varphi) \\
& X(\varphi, T)=\frac{m_{\text {Water }}}{m_{S G}}=X_{m}(T) \cdot f_{a}(c)
\end{aligned}
$$

$X_{m}(T)$ corresponds to the load of monomolecular layer of water on the silica gel surface. Since the water load of this layer is temperature dependent, the load at other relative humidity values is also. The function $f$ represents the sorption isotherm, which can be described with several formulas. The formula depends on the considered sorption effects, resulting in different levels of complexity. From simple approaches like described by Langmuir 
[2] to more detailed models like used by Brunauer et al. [3].

In a closed air volume, the adsorption or desorption of water, due to change in temperature, results in a change of absolute and relative humidity. This occurs, because the mass $\Delta m_{W V}$ of interchanged water vapor in the air volume must equal difference of adsorbed water on the silica gel surface:

$$
\begin{aligned}
& \Delta m_{W V}(\varphi, T)= \\
& m_{S G} \cdot\left[X\left(\varphi_{0}, T_{0}\right)-X(\varphi, T)\right]
\end{aligned}
$$

From (2) an implicit equation can be derived:

$$
\begin{aligned}
& c(T)=\frac{m_{W V}}{V_{c}}=c_{0}+\frac{m_{S G}}{V_{c}} \cdots \\
& \cdots\left[X_{m}\left(T_{0}\right) f_{a}\left(c_{0}\right)-X_{m}(T) f_{a}(c)\right]
\end{aligned}
$$

Equation (3) allows calculating the absolute humidity $\mathrm{C}$, with given start humidity $c_{0}$, start temperature $T_{0}$ and the sorption isotherm $f_{a}$. Obviously the ratio of the amount of silica gel to the volume of the air $V_{c}$ is important for the characteristic curve of the silica gel hygrostat. When a BET-isotherm is used, equation (3) cannot be solved analytically. To illustrate the influence of the silica gel mass, the change in dew point temperature was calculated in a numerical simulation. The effect caused by a heating $50 \mathrm{~K}$ is shown in Fig. 2 for different ratios auf silica gel mass to air volume.

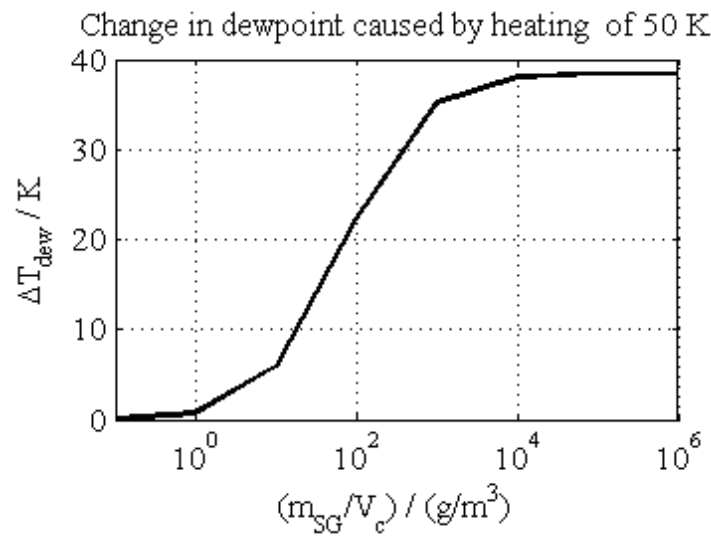

Fig. 2: Influence of silica gel mass on humidity change

As expected the achievable humidity change increases with a higher ratio of silica gel to chamber volume. But the potential of this is limited, due to the feedback of the sorption isotherm. This feedback follows from the fact, that the equilibrium is not only dependent on the temperature, but also on the air humidity.

Besides the silica gel mass, also the absolute humidity at $23^{\circ} \mathrm{C}\left(c_{0}\right)$ has an influence on the achievable change in dew point temperature (see Fig. 3). In a range typical for room climate this influence is not as distinct as the silica gel mass, but it is not negligible.

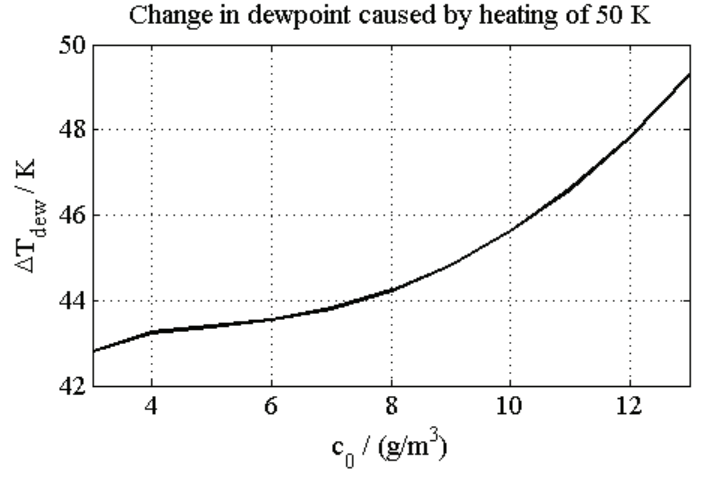

Fig. 3: Influence of hygrostat's start humidity

Between the calculated characteristic curves and the experimental determined ones, occurred several deviations. Possible reasons are readsorption processes at the chamber surface or an inhomogeneous temperature field in the silica gel bed. A temperature gradient in the bed would cause an adsorption of water in the cooler parts, which was desorbed in the warm parts. This makes clear, that there is the need for a homogeneous temperature field in the silica gel bed. Both explanations can be considered in simulations as a higher air volume in the hygrostat chamber (Fig. 4).

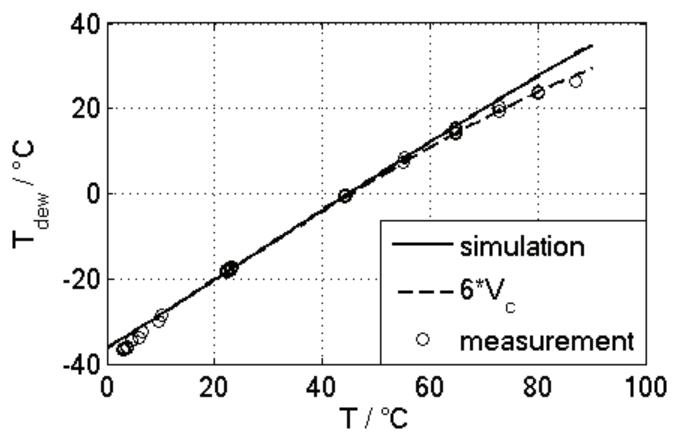

Fig. 4: Chracteristic curve of hygrostat with silica gel Type $M$

\section{Modelling of water transport}

The dynamic behavior of the silica gel hygrostat is influenced by the temperature controller of the bed and the water transport processes in 
the silica gel. A fast temperature control setup can be set up as described in the "Experimental setup"-section. Due to limited disposable power, the amount of silica gel and therefore also the air value should be as small as necessary.

The water transport consists of the transport phenomena in silica gel grain and the diffusion process in the air of the sorption bed. In a single grain there occurs water transport in the macroporous structures as well as in the microporous. In the smaller pores the mobility of the water molecules is limited along the body surface [4]. Therefore the dynamic behavior of water transport in the grain varies strongly in dependence of the pore structure. According to this structure very slow processes (e.g. surface diffusion) are superposed by fast processes (free diffusion in macro pores). This can be reflected in an overshoot of the step response of the hygrostat.

The dynamic behavior of the water transport between the silica gel grains mostly depends on the grain size and its distribution [5]. With growing grain size, a faster transport is expected. But in the examinations, which were performed in this work, the transport processes in the silica gel grain was dominant.

Another influence on the dynamic behavior is contributed by the curvature of the sorption isotherm [6]. In the shown application this effect increased, because the temperature dependent slope of the isotherms can be seen as an additional curvature. This is applicable, if the temperature changes in the mass transport process. A result of the additional curvature is a different dynamic behavior of desorption and adsorption processes, in which the desorption proceeds faster. In simulation this effect results directly out of the consideration of the temperature dependent slope and is also observed in measurement (see Fig. 5).

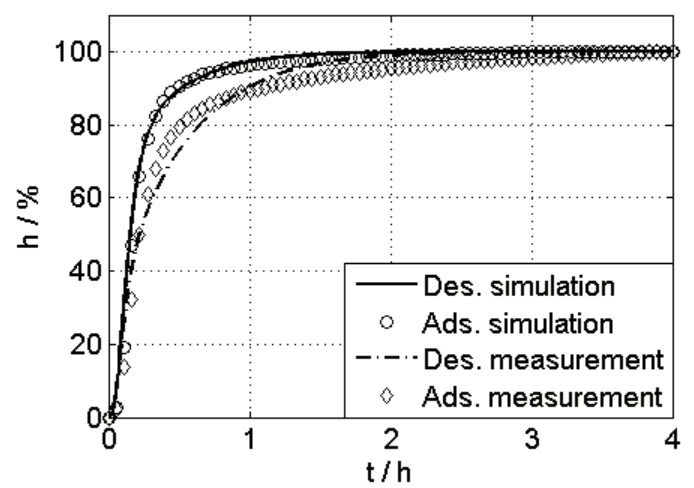

Fig. 5: normalized step response at sorption and desorption

\section{Experimental Setup}

A schematic of the experimental setup is shown in Fig. 6.

For fast temperature control and the possibility of cooling and heating, a thermoelectric cooler was used. For fast and homogeneous temperature control the silica gel was put into a heat sink (Fig. 7) attached to thermoelectric cooler (TEC). To reduce the heat transport from the silica gel bed to the air, the heat sink was surrounded by a thermal isolation layer.

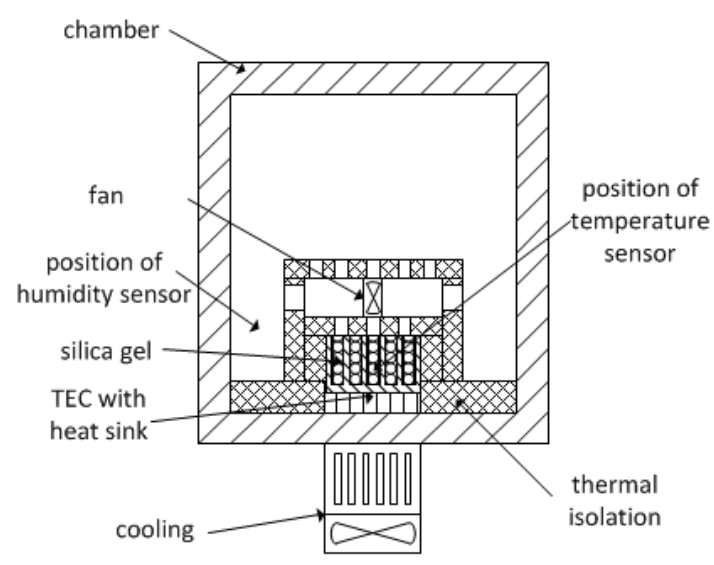

Fig. 6: Schematic of hygrostat chamber

To improve the dynamic behavior of moisture transport processes in the surrounding air, a fan was attached above the heat sink. Preliminary tests had shown that this setup improves the dynamic behavior of the whole hygrostat system.

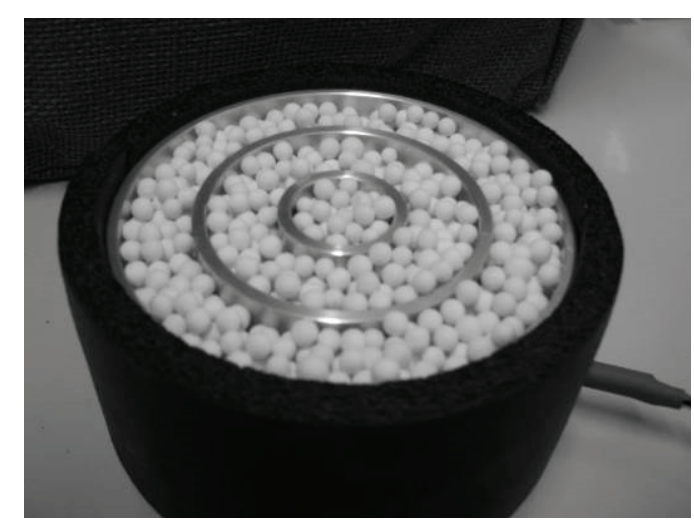

Fig. 7: Used heat sink with silica gel bed

The system was housed in a sealed chamber and the temperatures and relative humidity was determined with a sensor of type HYT271 supplied by Hygrosens [7]. By reason of the simultaneous measurement of temperature and relative humidity, other humidity characteristics could be calculated. Because of the independency from the air temperature, the dew point temperature was used to evaluate the hygrostat performance. 
In the experiments silica gel of type $\mathrm{M}$ was used with a grain size between $6 \mathrm{~mm}$ and $10 \mathrm{~mm}$. Additionally two kinds of silica gel type $\mathrm{E}$ were examined. The first was a granulate with grain sizes between $0.2 \mathrm{~mm}$ and $1 \mathrm{~mm}$ and second one was a globular sort with grain sizes between $2 \mathrm{~mm}$ and $5 \mathrm{~mm}$.

The working range of the hygrostat can be set up by controlling the silica gel temperature, while the chamber is open. After reaching the equilibrium state, the chamber is sealed and the temperature is controlled to room temperature. Depending on the previously temperature set point and the surrounding air humidity, the range is shifted relatively to the state without preconditioning.

\section{Results}

To evaluate the suitability of different types of silica gel, the gel was placed in the hygrostat and different silica gel temperatures were controlled in a range between $5^{\circ} \mathrm{C}$ and $60^{\circ} \mathrm{C}$. The change of humidity was observed in relation to the dew point temperature at $23^{\circ} \mathrm{C}$.

In Fig. 8 the influence of the pore type is shown. As one can see, there are only small deviations. These are probably produced by the higher density of the type $E$ grains, resulting in a higher silica gel mass of the completely filled bed.

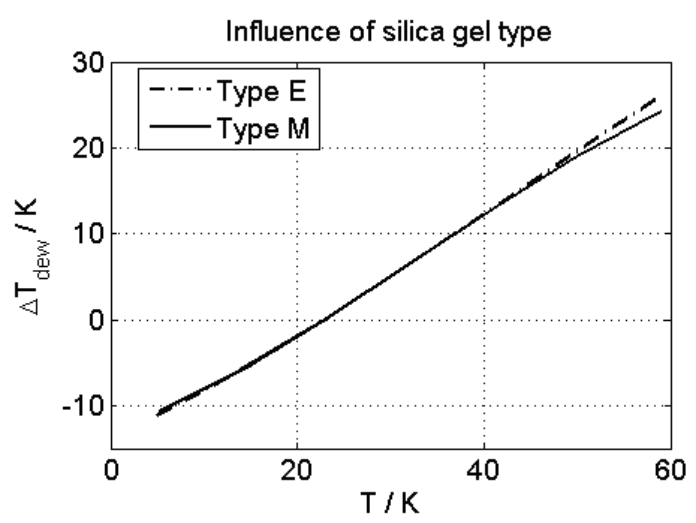

Fig. 8: characteristic curve of the hygrostat with different types of silica gel

To examine the impact of the grain size, the characteristic curves were also generated with the silica gel granulate and the globular sort, both of type $E$ (see Fig. 9). The globular silica gel allows a larger a change of dew point temperature, although the higher grain size. This shows a limitation of the improvement by reduction of grain size. The effect may be caused by transformations in the production process and should be subject of further investigations.

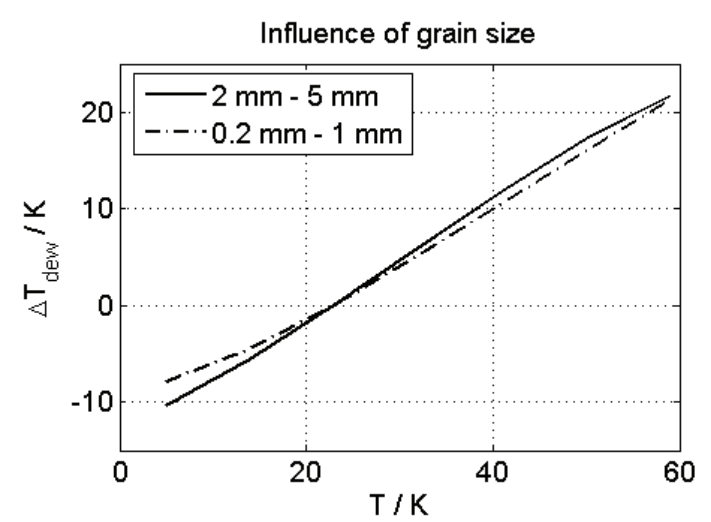

Fig. 9: characteristic curve of the hygrostat with different grain sizes

In further experiments the mass of the same sort of silica gel (type E, globular) was varied. This gives additional information about the sensitivity to changes in the fill level of the silica gel bed. Furthermore the influence of the silica gel package density can be estimated, which is not constant, when the type or grain size of silica gel is changed. As seen in Fig. 10, the impact is very low and occurs mostly at low levels of humidity. Given that it can be said, that the previously observed deviations are mostly caused by deviations in the pore structure and the grain surface.

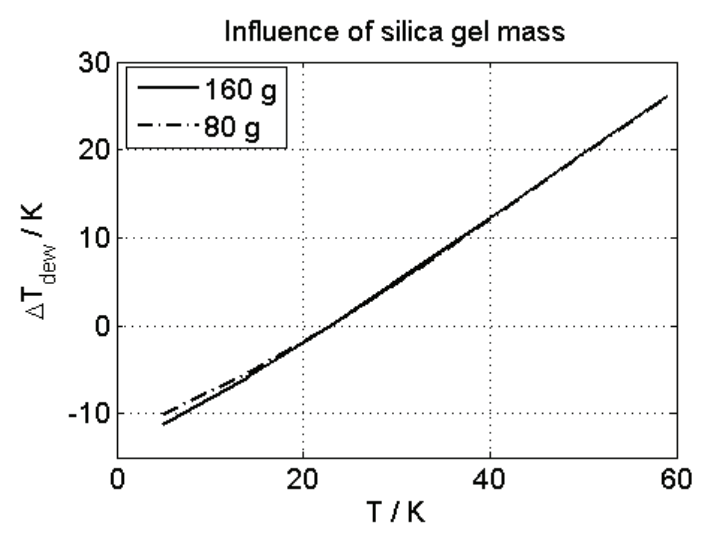

Fig. 10: characteristic curve of the hygrostat with different amounts of silica gel

Another important influence on the hygrostat characteristics is the working range. One factor is the nonlinear sorption isotherm, resulting in different equilibriums of the water load and humidity (see Fig. 3). But also the homogeneity of the temperature of the silica gel bed is important, because every area of different temperature has its own equilibrium point. In Fig. 11 there is shown the characteristic curve of granulate silica gel type $\mathrm{E}$ in a dew point temperature range between $-31^{\circ} \mathrm{C}$ and $1{ }^{\circ} \mathrm{C}$ and between $-22^{\circ} \mathrm{C}$ and $15^{\circ} \mathrm{C}$. For this sort of silica gel the effectiveness of the hygrostat is 
higher in a more humid working range. This coincides with the expected behavior.

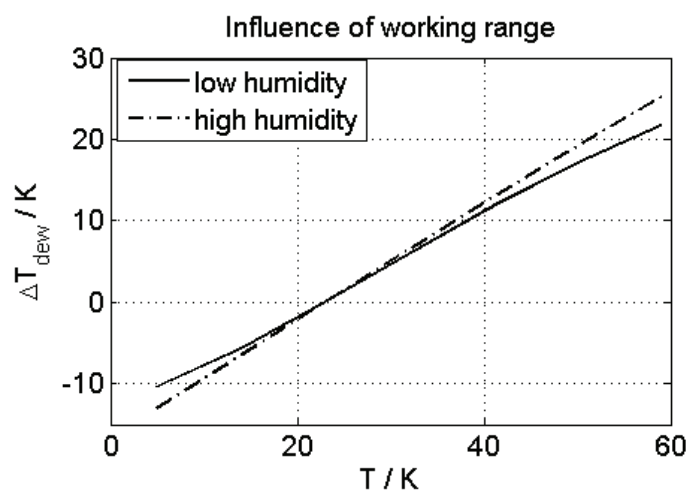

Fig. 11: characteristic curve of the hygrostat in different working ranges

\section{Conclusions}

The presented results imply to use as less silica gel as necessary, to achieve the desired change in humidity. A factor of safety for the silica gel mass could even worsen the effectiveness of the hygrostat. Also it was shown, that the maximum of this change is not only limited by the permitted temperature of the involved materials. But also it is limited by the properties of the silica gel. Within tested sorts of silica gel, the globular sort of type $E$ and medium grain size was most suitable.

To use the full capacity of the utilized silica gel, it is reasonable to use flat heat sink geometry to reduce the vertical thermal gradient in the bed. Also a proper thermal isolation of the bed is necessary.

\section{References}

[1] Fröhlich, Thomas; Hilbrunner, Falko: Vorrichtung und Verfahren zur Einstellung oder Regelung der Gasfeuchte in nahezu abgeschlossenen Räumen. Deutsche Patentanmeldung DE 10222438A1, 2003

[2] Langmuir, Irving: The evaporation, condensation and reflection of molecules and the mechanism of adsorption. In: Phys. Rev. 8, 1916, p. 149-176

[3] Brunauer, Stephen; Emmet, P.H.; Teller, Edward: Adsorption of Gases in Multimolecular Layers. In: J.Am.Chem.Soc. 60, Nr. 2, 1938, p. 309-319

[4] Busweiler, Ulrich: Nichtisotherme Ad- und Desorptionskinetik an Einzelkörnern technischer Adsorbentien am Beispiel der Wasserdampfsorption an Silicagel und Molekularsieb. Dissertation, 1984.

[5] Aristov, Yuriy I.; Glaznev, Ivan S.; Girnik, Ilya S.: Optimization of adsorption dynamics in adsorptive chillers: Loose grains configuration, Energy, 46 (1), pp. 484-492, 2012
[6] Kast, Werner: Adsorption aus der Gasphase: Ingenieurwissenschaftliche Grundlagen und technische Verfahren. Weinheim: VCH, 1988

[7] Innovative Sensor Technology: Hygrochip digital humidity sensor HYT-271. www.hygrochip.com/ fileadmin/user_upload/Produkte/Sensorelemente/ Digitale_Feuchtesensoren/HYT_271/IST_PDF/ EN_HYT̄271.pdf, 2011 